\title{
Quantitative Evaluation of Initial Symptoms as Predictors to Detect Adverse Drug Reactions Using Bayes' Theory: Expansion and Evaluation of Drug-Adverse Drug Reaction-Initial Symptom Combinations Using Adverse Event Reporting System Database
}

\author{
Daisuke Kobayashi, ${ }^{* a}$ Shigeru Hosaka, ${ }^{b}$ Emiko Inoue,${ }^{a}$ Kimie Ohshima, ${ }^{b}$ Nobuaki Kutsuma, ${ }^{b}$ \\ Shinji Oshima, ${ }^{a}$ and Yasushi Okuno, ${ }^{*}$ \\ ${ }^{a}$ Department of Analytical Pharmaceutics and Informatics, Faculty of Pharmaceutical Sciences, Josai University; \\ 1-1 Keyakidai, Sakado, Saitama 350-0295, Japan: ${ }^{b}$ Asahi-Chozai Co., Ltd.; 10F, JR Minami-shinjuku Bldg., Yoyogi, \\ Shibuya-ku, Tokyo 151-0053, Japan: and ${ }^{c}$ Department of Systems Biosciences for Drug Discovery, Graduate School \\ of Pharmaceutical Sciences, Kyoto University; Kyoto 606-8501, Japan. \\ Received November 19, 2012; accepted September 30, 2013
}

In prescription dispensing in Japan, to avoid adverse drug reactions (ADR) pharmacists provide patients with information concerning the initial symptoms (IS) of any ADR that might be caused by the drugs they have been prescribed. However, the usefulness of such information for preventing ADR has not been quantitatively evaluated. We previously performed a trial calculation of the usefulness of rash as a predictor of drug-induced liver disorders by applying Bayes' theorem and showed that the predictive utility of IS can be quantitatively evaluated using likelihood ratios. However, for other drug-ADR-IS combinations it was difficult to obtain the information required for the calculations from Japanese data alone. In this study, using the Adverse Event Reporting System (AERS) database of the U.S. Food and Drug Administration (FDA), we evaluated 132 drug-ADR-IS combinations that were considered to be potentially clinical significant. Regarding bezafibrate-associated rhabdomyolysis and cibenzoline-associated hypoglycemia, these ADR were not detected in cases involving monotherapy. For 58 combinations, no events that were considered to be IS of the target ADR developed. Fever, nausea, and decreased appetite were the IS of many ADR, making them very useful predictors. In contrast, pruritus and rash were not very useful. Fever might be a predictor of thiamazole-induced agranulocytosis or levofloxacin- or terbinafine-induced liver disorder, tremors might be useful for predicting paroxetine-induced serotonin syndrome, and decreased appetite might be a useful indicator of terbinafine-induced liver dysfunction.

Key words Bayes' theory; initial symptom; Adverse Event Reporting System; adverse drug reaction; information service

In Japan, when pharmacists dispense drugs to patients they provide information about any symptoms that the prescribed medication might induce that should cause the patient to consult a physician in order to avoid adverse drug reactions (ADR). Specifically, article 25-2 of the Pharmacists Act (1997) obliges pharmacists to describe to patients the initial symptoms (IS) of any ADR that might be induced by the drugs they have been prescribed.

Since the information provided about the IS of ADR has to be easy for patients to understand, less specific symptoms, mainly changes in their physical condition, such as fever, rash, or muscular pain, are described. However, it is doubtful whether such information is useful for detecting ADR.

In our previous report, we performed a trial calculation of the usefulness of rash as an IS of liver disorder; i.e., as an ADR predictor, using Bayes' theorem. ${ }^{1)}$ For voriconazole, the prior probability of liver disorder, 0.05 , was increased to 0.2 when a rash was present, whereas liver disorders only developed in 2 of 1000 patients who developed a rash after taking loxoprofen sodium, showing that the value of an IS as a predictor varies depending on the drug being taken.

Bayes' theorem is widely used to evaluate the predictability of a single or a combination of multiple diagnoses. ${ }^{2}$ However, to use Bayes' theorem to assess the utility of IS of ADR as diagnostic predictors, it is necessary to identify the probability of a specific symptom accompanying a particular ADR, and we were only able to determine these probabilities for a small number of drugs using Japanese data in our previous study.

The Adverse Event Reporting System (AERS) database, which is run by the U.S. Food and Drug Administration (FDA), contains over four million reports of adverse events (AE). It relies on reports of spontaneous adverse events being submitted to the FDA by health professionals, consumers, and manufacturers. In this study, we used this database in order to markedly increase the number of drug-ADR-IS combinations involving commonly used drugs for which the above mentioned calculations could be performed. In addition, the results of the calculations provided us with some interesting insights.

\section{THEORY}

Calculation of Posterior Probability When an IS (I) that is considered to be a predictor of a particular ADR (A) develops after a certain drug (D) is taken, the probability of A actually developing $\left(P_{\text {post }}\right)$ is given by the equation below:

$$
P_{\text {post }}=\frac{P_{\text {pre }}\left(Q_{\text {pre }}+Q_{n o n}\right)}{P_{\text {pre }}\left(Q_{\text {pre }}+Q_{\text {non }}\right)+P_{\text {other }}\left(Q_{\text {other }}+Q_{n o n}\right)+P_{\text {non }} Q_{\text {non }}}
$$




$$
\begin{aligned}
P_{\text {post }} & =\frac{P_{\text {pre }}\left(Q_{\text {pre }}+Q_{\text {non }}\right)}{P_{\text {pre }} Q_{\text {pre }}+P_{\text {other }} Q_{\text {other }}+Q_{\text {non }}\left(P_{\text {pre }}+P_{\text {other }}+P_{\text {non }}\right)} \\
& =\frac{P_{\text {pre }}\left(Q_{\text {pre }}+Q_{\text {non }}\right)}{Q_{t}-P_{\text {non }} Q_{\text {non }}+Q_{\text {non }}}
\end{aligned}
$$

where: $P_{\text {post }}$ : Incidence of A accompanied by I, $P_{\text {pre }}$ : Incidence of A, $P_{\text {other }}$ : Incidence of ADR other than A, $P_{\text {non }}$ : Probability of no ADR developing, $Q_{\text {pre }}$ : Probability of D-induced I accompanying A, $Q_{\text {other }}$ : Probability of D-induced I accompanying an ADR other than $\mathrm{A}, Q_{n o n}$ : Incidence of I-like symptoms that are not drug-related, $Q_{t}$ : Total incidence of D-induced I in clinical trials

$$
Q_{t}=P_{\text {pre }} Q_{\text {pre }}+P_{\text {other }} Q_{\text {other }}+P_{\text {non }} Q_{\text {non }}
$$

The addition of $Q_{\text {non }}$ to $Q_{\text {pre }}$ and $Q_{\text {other }}$ in Eq. 1 assumes that the incidence of I-like symptoms that are not drug-related is not included in $Q_{\text {pre }}$ or $Q_{\text {other }}$. ADR are defined as reactions for which an association with a particular drug cannot be ruled out, and IS are mild ADR. Accordingly, it can be assumed that $Q_{\text {non }}$ is not included in $Q_{\text {pre }}$ or $Q_{\text {other }}$.

$Q_{\text {pre }}$ and $Q_{\text {other }}$ were calculated using the AERS data. Since the AERS data are comprised of AE reports, $Q_{\text {pre }}$ and $Q_{\text {other }}$ were considered to include $Q_{\text {non }}$. However, since we calculated $Q_{\text {pre }}$ and $Q_{\text {other }}$ from the data of drugs used alone. Therefore, it is strongly assumed that reporters of AE considered the drug to be the primary suspect and reported the event as an ADR. Thus, in the calculation of $P_{\text {post }}$, we made 2 calculations: $Q_{n o n}$ was added to $Q_{\text {pre }}$ and $Q_{\text {other }}$ in one $(\mathrm{Cal}++)$ and not added in the other $(\mathrm{CalQO})$, broadening the range of interpretation of the results. The $P_{\text {post }}$ values produced using $\mathrm{Cal}++$ and CalQO are presented as $P_{\text {post }} Q+$ and $P_{\text {post }} Q 0$, respectively.

Equation 2 is only applicable when the total number of individuals who took a particular medication is known, as the latter figure is required $Q_{t}$. Since the AERS data did not describe the number of patients who took each drug without developing AE, we did not use Eq. 2 in this study.

Calculation of Likelihood Ratios The positive likelihood ratio was calculated using Eq. 3.

$$
L R+=\frac{O d_{\text {post }}}{O d_{\text {pre }}}=\frac{P_{\text {post }}\left(1-P_{\text {pre }}\right)}{P_{\text {pre }}\left(1-P_{\text {post }}\right)}
$$

where: $L R+$ : Likelihood ratio, $O d_{\text {pre }}$ : Prior odds, $O d_{\text {post }}$ : Posterior odds

$L R+$ values that were calculated using $P_{\text {post }} Q+$ and $P_{\text {post }} Q 0$ as $P_{\text {post }}$ were designated as $L R+Q+$ and $L R+Q 0$, respectively.

\section{METHODS}

Incidence of IS-Like Symptoms That Were Not Caused by the Target Drug $\left(\boldsymbol{Q}_{n o n}\right)$ The IS of ADR, such as fever, headache, and muscular pain, are also experienced as changes in physical condition that are not caused by drugs in daily life. Of these changes in physical condition, those that occur without an obvious cause, such as a cold or excess exercise, need to be differentiated from the IS of ADR, and knowledge about their incidence is required to calculate the frequency of non-specific background IS-like symptoms; i.e., $Q_{\text {non }}$ in Eq. 1 . Thus, we first investigated $Q_{n o n}$.

Selection of IS for the $\boldsymbol{Q}_{\text {non }}$ Investigation The examined IS were selected from "the Manual for Handling Disorders due to Adverse Drug Reactions" (MHD). ${ }^{3}$ The MHD was published by the Ministry of Health, Labour, and Welfare and was produced in cooperation with academics in order to develop a strategy for dealing with disorders caused by ADR. In particular, the MHD aims to prevent severe ADR being overlooked during their early stages. ${ }^{4}$ So, we selected IS that exhibited high incidences from the MHD for our investigation of $Q_{\text {non }}$.

Survey of $\boldsymbol{Q}_{\text {non }} Q_{\text {non }}$ was investigated using an internetbased questionnaire developed by Rakuten Research (Rakuten Research, Inc., Shinagawa, Tokyo, Japan). Nine hundred males and 900 females belonging to 6 decile age groups, ranging from subjects in their 20 s to patients in their 70 s (150 males and 150 females in each age group), were surveyed about any changes in their physical condition of unknown cause that had occurred during the last 3 months. The survey period was set at 3 months because many ADR develop within 3 months of the initiation of drug treatment. ${ }^{5)}$ We have reported the findings of this survey previously.1) The results are shown together with the incidence of urticaria-like changes in physical condition in Table 1.

Selection of Drug-ADR-IS Combinations On the condition that one of the target IS examined in the investigation of $Q_{n o n}$ was included in each combination, 3 pharmacists selected drug-ADR-IS combinations from the MHD from the viewpoint of clinical importance about patient compliance instruction. That is, the process used to select the drug-ADRIS combinations was as follows: First, IS that were frequently mentioned in the MHD were extracted. Then, we identified ADR involving these IS. Finally, we extracted drugs associated with these ADR.

The terms used to describe the ADR and IS were standardized to the Preferred Terms outlined in the Japanese edition of the ICH Medical Dictionary for Regulatory Activities Terminology $(\mathrm{MedDR} / \mathrm{J})$.

Data Collection. $\boldsymbol{P}_{\text {pre }}, \boldsymbol{P}_{\text {other }}$, and $\boldsymbol{P}_{\text {non }}$ The probabilities of the target ADR $\left(P_{\text {pre }}\right)$, ADR other than the target $\left(P_{\text {other }}\right)$, and no ADR developing $\left(P_{n o n}\right)$ were adopted from the "ADR frequencies classified by item and a list of abnormal clinical laboratory test results" (a list of ADR) section of the postmarketing surveillance (PMS) data on each drug's interview form (IF). The IF of brand name drugs were used.

$\boldsymbol{Q}_{\text {pre }}$ and $\boldsymbol{Q}_{\text {other }}$ Using the AERS data, the total number of cases of ADR, the number of cases of the target ADR, and the number of cases of the target ADR that were accompanied by the target IS were investigated for each drug used alone, and $Q_{\text {pre }}$ and $Q_{\text {other }}$ were determined.

Statistical Analysis The difference between $P_{\text {pre }}$ and $P_{\text {post }}$ was analyzed arbitrarily by setting the population size to that of the PMS.

\section{RESULTS}

Drug-ADR-IS (D-A-I) Combinations and the Results of the AERS Survey The target D-A-I combinations are shown in Table 2. " $\star$ " represents IS that were not found in the AERS database.

Regarding bezafibrate-associatefd rhabdomyolysis and cibenzoline-associated hypoglycemia, the ADR were not induced by these drugs alone. Therefore, they have been marked with " $\star \star \star . "$ 
Table 1. Incidence Rates of Changes in Body Condition

\begin{tabular}{|c|c|c|c|c|c|}
\hline ID & Symptom & Incidence & ID & Symptom & Incidence \\
\hline 1 & Fatigue & 0.1644 & 27 & Increased blood pressure & 0.0494 \\
\hline 2 & Pruritus & 0.1450 & 28 & Dry cough & 0.0494 \\
\hline 3 & Malaise & 0.1339 & 29 & Edema & 0.0439 \\
\hline 4 & Headache & 0.1250 & 30 & Nausea & 0.0428 \\
\hline 5 & Diarrhea & 0.1161 & 31 & Anemia & 0.0417 \\
\hline 6 & Arthralgia & 0.1139 & 32 & Musculoskeletal stiffness & 0.0411 \\
\hline 7 & Tinnitus & 0.1000 & 33 & Hyperhidrosis & 0.0406 \\
\hline 8 & Abdominal distensiom & 0.0961 & 34 & Erythema & 0.0389 \\
\hline 9 & Constipation & 0.0883 & 35 & Wet cough & 0.0389 \\
\hline 10 & Somnolence & 0.0872 & 36 & Decreased appetite & 0.0378 \\
\hline 11 & Dizziness & 0.0861 & 37 & Pyrexia & 0.0350 \\
\hline 12 & Rash & 0.0833 & 38 & Facial edema & 0.0294 \\
\hline 13 & Insomnia & 0.0800 & 39 & Tachycardia & 0.0289 \\
\hline 14 & Pollakiuria & 0.0783 & 40 & Dysponea & 0.0250 \\
\hline 15 & Oropharyngeal pain & 0.0783 & 41 & Bruising & 0.0239 \\
\hline 16 & Abdominal pain & 0.0761 & 42 & Black stools & 0.0228 \\
\hline 17 & Abdominal discomfort & 0.0700 & 43 & Dyslalia & 0.0206 \\
\hline 18 & Urticaria & 0.0614 & 44 & Asthenia & 0.0200 \\
\hline 19 & Palpitations & 0.0606 & 45 & Tremors & 0.0194 \\
\hline 20 & Muscle ache & 0.0572 & 46 & Oliguria & 0.0189 \\
\hline 21 & Epigastrial pain & 0.0561 & 47 & Vomiting & 0.0189 \\
\hline 22 & Hemorrhage $(*)$ & 0.0556 & 48 & Wheezing & 0.0161 \\
\hline 23 & Thirst & 0.0544 & 49 & Ageusia & 0.0150 \\
\hline 24 & Heart burn & 0.0528 & 50 & Oral hypoesthesia & 0.0078 \\
\hline 25 & Chest pain & 0.0517 & 51 & Hematuria & 0.0078 \\
\hline 26 & Hypesthesia & 0.0517 & & & \\
\hline
\end{tabular}

(*) Subcutaneous hemorrhaging + gingival bleeding + epistaxis.

Sixty-two of the 132 combinations were not marked with " $\star$ " or " $\star \star \star$ " and were subjected to the calculation.

CalQ0 and CalQ+ The results of the survey and the calculations for the 62 combinations are shown in Table 3.

A simplified list of the $L R+Q 0$ and $L R+Q+$ calculation results, as well as drug names and fully spelt out descriptions of the ADR/AE and IS are shown in Table 4.

For Ti-Ld-Py, which was ranked in first place, $P_{\text {post }} Q+$ was not calculated because $Q_{\text {pre }}+Q_{\text {non }}$ exceeded 1 .

The combinations are listed in decreasing order of $L R+Q 0$ in Tables 3 and 4. Their ranks were similar to those based on $L R+Q+$ (rank correlation coefficient: 0.993, calculated excluding Ti-Ld-Py). Thirty combinations had $L R+Q 0$ values of less than 1; i.e., their $P_{\text {post }}$ values were smaller than their $P_{p r e}$ values. This was due to the fact that their $Q_{\text {pre }}$ values were lower than their associated $Q_{\text {non }}$ values, indicating that the incidence of IS-like changes in physical condition that occur during daily life and are unrelated to the medication was higher than the incidence of drug-induced IS; therefore, it would be inappropriate to regard the target symptoms as IS of ADR. Although the $L R+Q+$ values of some combinations exceeded 1 , most of these combinations exhibited insignificant differences between their $P_{\text {pre }}$ and $P_{\text {post }} Q^{+}$values, and the associated symptoms were demonstrated to be inappropriate for detecting the target $\mathrm{ADR}$, as was found in the $L R+Q 0$-based evaluation.

Usefulness of IS Development of the IS marked with " $\star$ " in Table 2 was not described in ADR cases of the drugs, suggesting low-usefulness as predictors. Provision of the information of these IS are not only less beneficial but also may induce only a nocebo effect, to which attention should be paid. ${ }^{6)}$

The combinations for which $P_{\text {post }}$ was calculable (Table 3) are summarized according to IS in Table 5. Combinations for which both $P_{\text {post }} Q 0$ and $P_{\text {post }} Q+$ were significantly greater than $P_{\text {pre }}$ and $L R+Q 0$ was greater than 1 were regarded as useful.

As a result, pyrexia was found to be useful in 7 of 10 drugADR-IS combinations for which the parameters could be calculated, suggesting its usefulness as an IS. Vomiting (4/5) and decreased appetite (3/6) were also suggested to be useful ADR predictors. Pruritus $(0 / 11)$ and rash $(0 / 9)$ are widely informed as IS, but were not found to be useful in any of the drugADR-IS combinations examined in this study. However, although pyrexia was considered to be useful, it did not develop in 5 of 15 candidate drug-ADR-IS combinations, which were marked with " $\star$," demonstrating that the type of IS induced and the likelihood of IS developing differ depending on the type of drug and ADR.

To prevent thiamazole-induced agranulocytosis, which was ranked highly in Tables 3 and 4, we suggest that fever should be selected as the IS, whereas paroxetine-induced serotonin syndrome can be detected by selecting tremors, levofloxacin- or terbinafine-induced liver disorder can be predicted by selecting fever, and decreased appetite might be a useful indicator of terbinafine-induced liver dysfunction. All of these IS were selected because they exhibited $L R+$ values of greater than 5.0. However, the calculations for fever for ticlopidineinduced liver disorder and decreased appetite for acarboseinduced liver dysfunction may have been less reliable because 
Table 2. Selected Drug-ADR-IS Combinations and Results of an AERS Database Search

\begin{tabular}{|c|c|c|c|c|}
\hline ID & Drg & AR & IS & Drug-ADR-IS, abbreviation \\
\hline 1 & Acarbose & Abnormal hepatic function & Decreased appetite & Ac-Hf-Da \\
\hline 2 & Acarbose & Abnormal hepatic function & Malaise $\star$ & Ac-Hf-Ml \\
\hline 3 & Acarbose & Abnormal hepatic function & Nausea $\star$ & Ac-Hf-Na \\
\hline 4 & Acarbose & Abnormal hepatic function & Pruritus $\star$ & Ac-Hf-Pr \\
\hline 5 & Acarbose & Abnormal hepatic function & Pyrexia $\star$ & Ac-Hf-Py \\
\hline 6 & Acarbose & Abnormal hepatic function & $\operatorname{Rash} \star$ & Ac-Hf-Ra \\
\hline 7 & Acarbose & Abnormal hepatic function & Vomiting $\star$ & Ac-Hf-Vo \\
\hline 8 & Acarbose & Liver disorder & Decreased appetite $\star$ & Ac-Ld-Da \\
\hline 9 & Acarbose & Liver disorder & Malaise $\star$ & Ac-Ld-Ml \\
\hline 10 & Acarbose & Liver disorder & Nausea $\star$ & Ac-Ld-Na \\
\hline 11 & Acarbose & Liver disorder & Pruritus $\star$ & Ac-Ld-Pr \\
\hline 12 & Acarbose & Liver disorder & Pyrexia $\star$ & Ac-Ld-Py \\
\hline 13 & Acarbose & Liver disorder & $\operatorname{Rash} \star$ & Ac-Ld-Ra \\
\hline 14 & Acarbose & Liver disorder & Vomiting $\star$ & Ac-Ld-Vo \\
\hline 15 & Atorvastatin calcium hydrate & Abnormal hepatic function & Decreased appetite & At-Hf-Da \\
\hline 16 & Atorvastatin calcium hydrate & Abnormal hepatic function & Malaise & At-Hf-Ml \\
\hline 17 & Atorvastatin calcium hydrate & Abnormal hepatic function & Nausea & At-Hf-Na \\
\hline 18 & Atorvastatin calcium hydrate & Abnormal hepatic function & Pruritus & At-Hf-Pr \\
\hline 19 & Atorvastatin calcium hydrate & Abnormal hepatic function & Pyrexia & At-Hf-Py \\
\hline 20 & Atorvastatin calcium hydrate & Abnormal hepatic function & Rash & At-Hf-Ra \\
\hline 21 & Atorvastatin calcium hydrate & Abnormal hepatic function & Vomiting & At-Hf-Vo \\
\hline 22 & Atorvastatin calcium hydrate & Liver disorder & Decreased appetite & At-Ld-Da \\
\hline 23 & Atorvastatin calcium hydrate & Liver disorder & Malaise & At-Ld-Ml \\
\hline 24 & Atorvastatin calcium hydrate & Liver disorder & Nausea & At-Ld-Na \\
\hline 25 & Atorvastatin calcium hydrate & Liver disorder & Pruritus $\star$ & At-Ld-Pr \\
\hline 26 & Atorvastatin calcium hydrate & Liver disorder & Pyrexia & At-Ld-Py \\
\hline 27 & Atorvastatin calcium hydrate & Liver disorder & $\operatorname{Rash} \star$ & At-Ld-Ra \\
\hline 28 & Atorvastatin calcium hydrate & Liver disorder & Vomiting & At-Ld-Vo \\
\hline 29 & Bezafibrate & Rhabdomyolysis $\star \star$ & Asthenia & Be-Rh-As \\
\hline 30 & Bezafibrate & Rhabdomyolysis $\star \star$ & Hematuria & $\mathrm{Be}-\mathrm{Rh}-\mathrm{Hu}$ \\
\hline 31 & Bezafibrate & Rhabdomyolysis $\star \star \star$ & Malaise & Be-Rh-Ml \\
\hline 32 & Bezafibrate & Rhabdomyolysis $\star \star$ & Myalgia & Be-Rh-My \\
\hline 33 & Cibenzoline succinate & Hypoglycemia $\star \star \star$ & Asthenia & Ci-Hy-As \\
\hline 34 & Cibenzoline succinate & Hypoglycemia $\star \star \star$ & Dizziness & Ci-Hy-Dz \\
\hline 35 & Cibenzoline succinate & Hypoglycemia $\star \star$ & Dyslalia & Ci-Hy-Dl \\
\hline 36 & Cibenzoline succinate & Hypoglycemia $\star \star \star$ & Headache & $\mathrm{Ci}-\mathrm{Hy}-\mathrm{Hd}$ \\
\hline 37 & Cibenzoline succinate & Hypoglycemia $\star \star \star$ & Nausea & $\mathrm{Ci}-\mathrm{Hy}-\mathrm{Na}$ \\
\hline 38 & Cibenzoline succinate & Hypoglycemia $\star \star \star$ & Palpitations & $\mathrm{Ci}-\mathrm{Hy}-\mathrm{Pa}$ \\
\hline 39 & Cibenzoline succinate & Hypoglycemia $\star \star \star$ & Somnolence & $\mathrm{Ci}-\mathrm{Hy}-\mathrm{So}$ \\
\hline 40 & Cibenzoline succinate & Hypoglycemia $\star \star \star$ & Tremors & $\mathrm{Ci}-\mathrm{Hy}-\mathrm{Tr}$ \\
\hline 41 & Famotidine & Neutropenia & Oropharyngeal pain $\star$ & Fa-Np-Or \\
\hline 42 & Famotidine & Neutropenia & Pyrexia $\star$ & Fa-Np-Py \\
\hline 43 & Glimepiride & Hypoglycemia & Asthenia & Gl-Hy-As \\
\hline 44 & Glimepiride & Hypoglycemia & Dizziness & Gl-Hy-Dz \\
\hline 45 & Glimepiride & Hypoglycemia & Dyslalia $\star$ & Gl-Hy-Dl \\
\hline 46 & Glimepiride & Hypoglycemia & Headache $\star$ & Gl-Hy-Hd \\
\hline 47 & Glimepiride & Hypoglycemia & Nausea $\star$ & Gl-Hy-Na \\
\hline 48 & Glimepiride & Hypoglycemia & Palpitations $\star$ & Gl-Hy-Pa \\
\hline 49 & Glimepiride & Hypoglycemia & Somnolence $\star$ & Gl-Hy-So \\
\hline 50 & Glimepiride & Hypoglycemia & Tremors & Gl-Hy-Tr \\
\hline 51 & Levofloxacin hydrate & Anaphylactoid reaction & Dyspnoea & Le-Ar-Dn \\
\hline 52 & Levofloxacin hydrate & Anaphylactoid reaction & Palpitations $\star$ & Le-Ar-Pa \\
\hline 53 & Levofloxacin hydrate & Anaphylactoid reaction & Pruritus $\star$ & Le-Ar-Pr \\
\hline 54 & Levofloxacin hydrate & Anaphylactoid reaction & Urticaria & Le-Ar-Ur \\
\hline 55 & Levofloxacin hydrate & Anaphylactoid shock & Dyspnoea & Le-As-Dn \\
\hline 56 & Levofloxacin hydrate & Anaphylactoid shock & Palpitations $\star$ & Le-As-Pa \\
\hline 57 & Levofloxacin hydrate & Anaphylactoid shock & Pruritus $\star$ & Le-As-Pr \\
\hline 58 & Levofloxacin hydrate & Anaphylactoid shock & Urticaria & Le-As-Ur \\
\hline 59 & Levofloxacin hydrate & Abnormal hepatic function & Decreased appetite & Le-Hf-Da \\
\hline 60 & Levofloxacin hydrate & Abnormal hepatic function & Malaise & Le-Hf-Ml \\
\hline 61 & Levofloxacin hydrate & Abnormal hepatic function & Nausea $\star$ & Le-Hf-Na \\
\hline 62 & Levofloxacin hydrate & Abnormal hepatic function & Pruritus $\star$ & Le-Hf-Pr \\
\hline 63 & Levofloxacin hydrate & Abnormal hepatic function & Pyrexia & Le-Hf-Py \\
\hline 64 & Levofloxacin hydrate & Abnormal hepatic function & Rash & Le-Hf-Ra \\
\hline 65 & Levofloxacin hydrate & Abnormal hepatic function & Vomiting $\star$ & Le-Hf-Vo \\
\hline 66 & Levofloxacin hydrate & Liver disorder & Decreased appetite $\star$ & Le-Ld-Da \\
\hline 67 & Levofloxacin hydrate & Liver disorder & Malaise & Le-Ld-Ml \\
\hline
\end{tabular}


Table 2. Continued

\begin{tabular}{|c|c|c|c|c|}
\hline ID & Drg & AR & IS & Drug-ADR-IS, abbreviation \\
\hline 68 & Levofloxacin hydrate & Liver disorder & Nausea & Le-Ld-Na \\
\hline 69 & Levofloxacin hydrate & Liver disorder & Pruritus $\star$ & Le-Ld-Pr \\
\hline 70 & Levofloxacin hydrate & Liver disorder & Pyrexia & Le-Ld-Py \\
\hline 71 & Levofloxacin hydrate & Liver disorder & $\operatorname{Rash} \star$ & Le-Ld-Ra \\
\hline 72 & Levofloxacin hydrate & Liver disorder & Vomiting & Le-Ld-Vo \\
\hline 73 & Paroxetine hydrochloride hydrate & Serotonin syndrome & Diarrhea & Pa-Ss-Dr \\
\hline 74 & Paroxetine hydrochloride hydrate & Serotonin syndrome & Hyperhidrosis & Pa-Ss-Hh \\
\hline 75 & Paroxetine hydrochloride hydrate & Serotonin syndrome & Pyrexia & Pa-Ss-Py \\
\hline 76 & Paroxetine hydrochloride hydrate & Serotonin syndrome & Tachycardia & Pa-Ss-Ta \\
\hline 77 & Paroxetine hydrochloride hydrate & Serotonin syndrome & Tremors & $\mathrm{Pa}-\mathrm{Ss}-\mathrm{Tr}$ \\
\hline 78 & Pioglitazone hydrochloride & Congestive heart failure & Dyspnoea & Pi-Cf-Dn \\
\hline 79 & Pioglitazone hydrochloride & Congestive heart failure & Fatigue & Pi-Cf-Fg \\
\hline 80 & Pioglitazone hydrochloride & Congestive heart failure & Edema & Pi-Cf-Oe \\
\hline 81 & Raloxifene hydrochloride & Deep vein thrombosis & Chest pain & Ra-Dt-Cp \\
\hline 82 & Raloxifene hydrochloride & Deep vein thrombosis & Dyslalia $\star$ & Ra-Dt-Dl \\
\hline 83 & Raloxifene hydrochloride & Deep vein thrombosis & Dyspnoea & Ra-Dt-Dn \\
\hline 84 & Raloxifene hydrochloride & Venous thrombosis & Chest pain $\star$ & $\mathrm{Ra}-\mathrm{Vt}-\mathrm{Cp}$ \\
\hline 85 & Raloxifene hydrochloride & Venous thrombosis & Dyslalia $\star$ & Ra-Vt-Dl \\
\hline 86 & Raloxifene hydrochloride & Venous thrombosis & Dyspnoea $\star$ & Ra-Vt-Dn \\
\hline 87 & Risperidone & Neuroleptic malignant syndrome & Increased blood pressure & Ri-Nm-Bi \\
\hline 88 & Risperidone & Neuroleptic malignant syndrome & Dyslalia & Ri-Nm-Dl \\
\hline 89 & Risperidone & Neuroleptic malignant syndrome & Hyperhidrosis & Ri-Nm-Hh \\
\hline 90 & Risperidone & Neuroleptic malignant syndrome & Musculoskeletal stiffness & Ri-Nm-Ms \\
\hline 91 & Risperidone & Neuroleptic malignant syndrome & Pyrexia & Ri-Nm-Py \\
\hline 92 & Risperidone & Neuroleptic malignant syndrome & Tachycardia & Ri-Nm-Ta \\
\hline 93 & Risperidone & Neuroleptic malignant syndrome & Tremors & Ri-Nm-Tr \\
\hline 94 & Terbinafine hydrochloride & Abnormal hepatic function & Decreased appetite & Te-Hf-Da \\
\hline 95 & Terbinafine hydrochloride & Abnormal hepatic function & Malaise & Te-Hf-Ml \\
\hline 96 & Terbinafine hydrochloride & Abnormal hepatic function & Nausea & Te-Hf-Na \\
\hline 97 & Terbinafine hydrochloride & Abnormal hepatic function & Pruritus & Te-Hf-Pr \\
\hline 98 & Terbinafine hydrochloride & Abnormal hepatic function & Pyrexia & Te-Hf-Py \\
\hline 99 & Terbinafine hydrochloride & Abnormal hepatic function & $\operatorname{Rash} \star$ & Te-Hf-Ra \\
\hline 100 & Terbinafine hydrochloride & Abnormal hepatic function & Vomiting & Te-Hf-Vo \\
\hline 101 & Terbinafine hydrochloride & Liver disorder & Decreased appetite & Te-Ld-Da \\
\hline 102 & Terbinafine hydrochloride & Liver disorder & Malaise & Te-Ld-Ml \\
\hline 103 & Terbinafine hydrochloride & Liver disorder & Nausea & Te-Ld-Na \\
\hline 104 & Terbinafine hydrochloride & Liver disorder & Pruritus & Te-Ld-Pr \\
\hline 105 & Terbinafine hydrochloride & Liver disorder & Pyrexia & Te-Ld-Py \\
\hline 106 & Terbinafine hydrochloride & Liver disorder & Rash & Te-Ld-Ra \\
\hline 107 & Terbinafine hydrochloride & Liver disorder & Vomiting & Te-Ld-Vo \\
\hline 108 & Thiamazole & Agranulocytosis & Oropharyngeal pain & Th-Ag-Or \\
\hline 109 & Thiamazole & Agranulocytosis & Pyrexia & Th-Ag-Py \\
\hline 110 & Ticlopidine hydrochloride & Anemia & Fatigue $\star$ & Ti-An-Fg \\
\hline 111 & Ticlopidine hydrochloride & Anemia & Headache & Ti-An-Hd \\
\hline 112 & Ticlopidine hydrochloride & Anemia & Malaise $\star \star$ & Ti-An-Ml \\
\hline 113 & Ticlopidine hydrochloride & Anemia & Palpitations $\star$ & Ti-An-Pa \\
\hline 114 & Ticlopidine hydrochloride & Cerebral hemorrhage & Hemorrhage $(*) \star$ & Ti-Ch-Hr \\
\hline 115 & Ticlopidine hydrochloride & Granulocytopenia & Oropharyngeal pain $\star$ & Ti-Gp-Or \\
\hline 116 & Ticlopidine hydrochloride & Granulocytopenia & Pyrexia $\star$ & Ti-Gp-Py \\
\hline 117 & Ticlopidine hydrochloride & Liver disorder & Decreased appetite $\star$ & Ti-Ld-Da \\
\hline 118 & Ticlopidine hydrochloride & Liver disorder & Malaise $\star$ & Ti-Ld-Ml \\
\hline 119 & Ticlopidine hydrochloride & Liver disorder & Nausea $\star$ & Ti-Ld-Na \\
\hline 120 & Ticlopidine hydrochloride & Liver disorder & Pruritus $\star$ & Ti-Ld-Pr \\
\hline 121 & Ticlopidine hydrochloride & Liver disorder & Pyrexia & Ti-Ld-Py \\
\hline 122 & Ticlopidine hydrochloride & Liver disorder & $\operatorname{Rash} \star$ & Ti-Ld-Ra \\
\hline 123 & Ticlopidine hydrochloride & Liver disorder & Vomiting $\star$ & Ti-Ld-Vo \\
\hline 124 & Ticlopidine hydrochloride & Pancytopenia & Dizziness $\star$ & Ti-Pp-Dz \\
\hline 125 & Ticlopidine hydrochloride & Pancytopenia & Dyspnoea $\star$ & Ti-Pp-Dn \\
\hline 126 & Ticlopidine hydrochloride & Pancytopenia & Fatigue $\star$ & Ti-Pp-Fg \\
\hline 127 & Ticlopidine hydrochloride & Pancytopenia & Hematuria $\star$ & Ti-Pp-Hu \\
\hline 128 & Ticlopidine hydrochloride & Pancytopenia & Hemorrhage $(*) \star$ & Ti-Pp-Hr \\
\hline 129 & Ticlopidine hydrochloride & Pancytopenia & Oropharyngeal pain $\star$ & Ti-Pp-Or \\
\hline 130 & Ticlopidine hydrochloride & Pancytopenia & Palpitations $\star \star$ & Ti-Pp-Pa \\
\hline 131 & Ticlopidine hydrochloride & Pancytopenia & Pyrexia $\star$ & Ti-Pp-Py \\
\hline 132 & Ticlopidine hydrochloride & Thrombocytopenia & Hemorrhage $(*) \star$ & Ti-Tp-Hr \\
\hline
\end{tabular}

(*) Subcutaneous hemorrhaging + gingival bleeding+epistaxis. $\star$ This IS was not reported. $\star \star$ This ADR was not reported in cases involving monotherapy. 


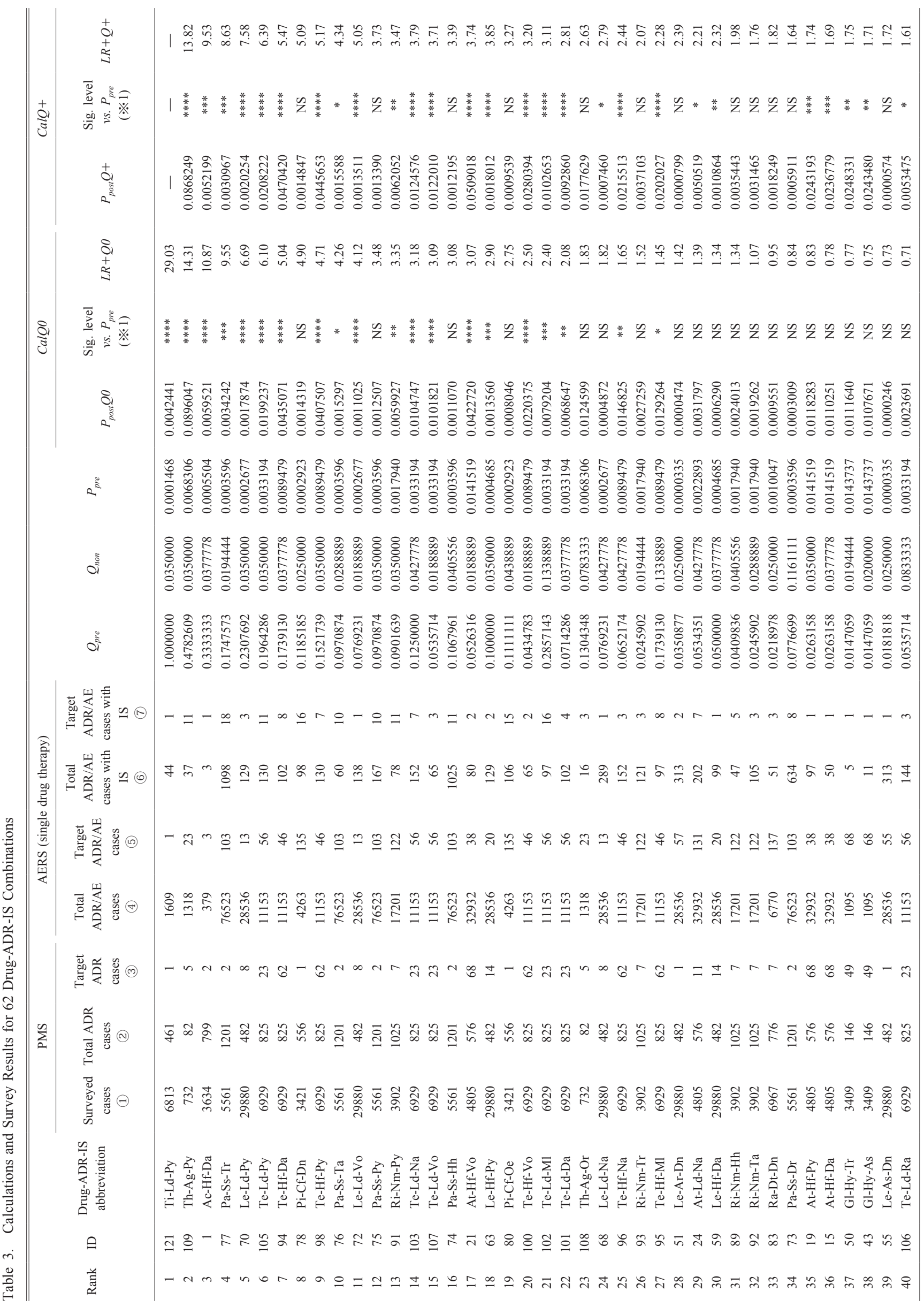




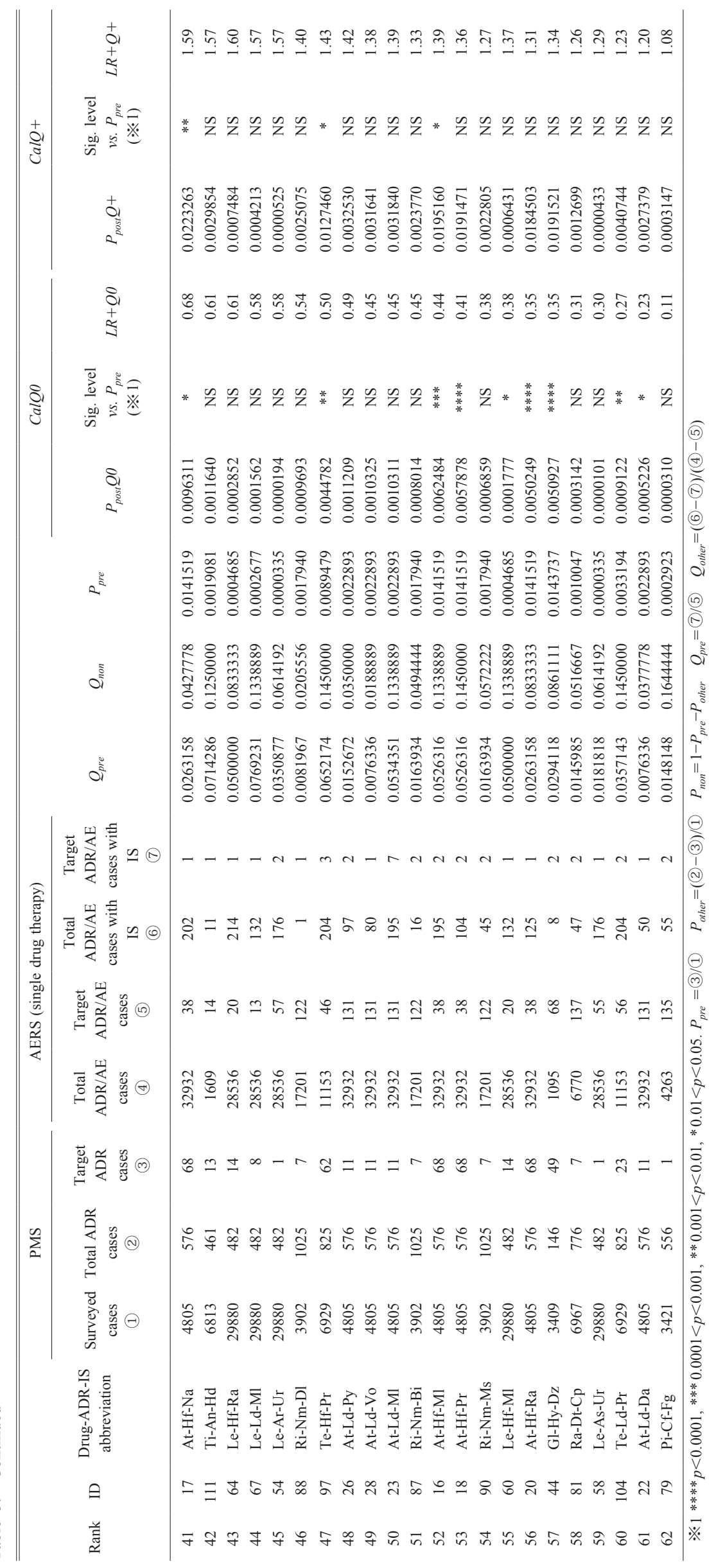




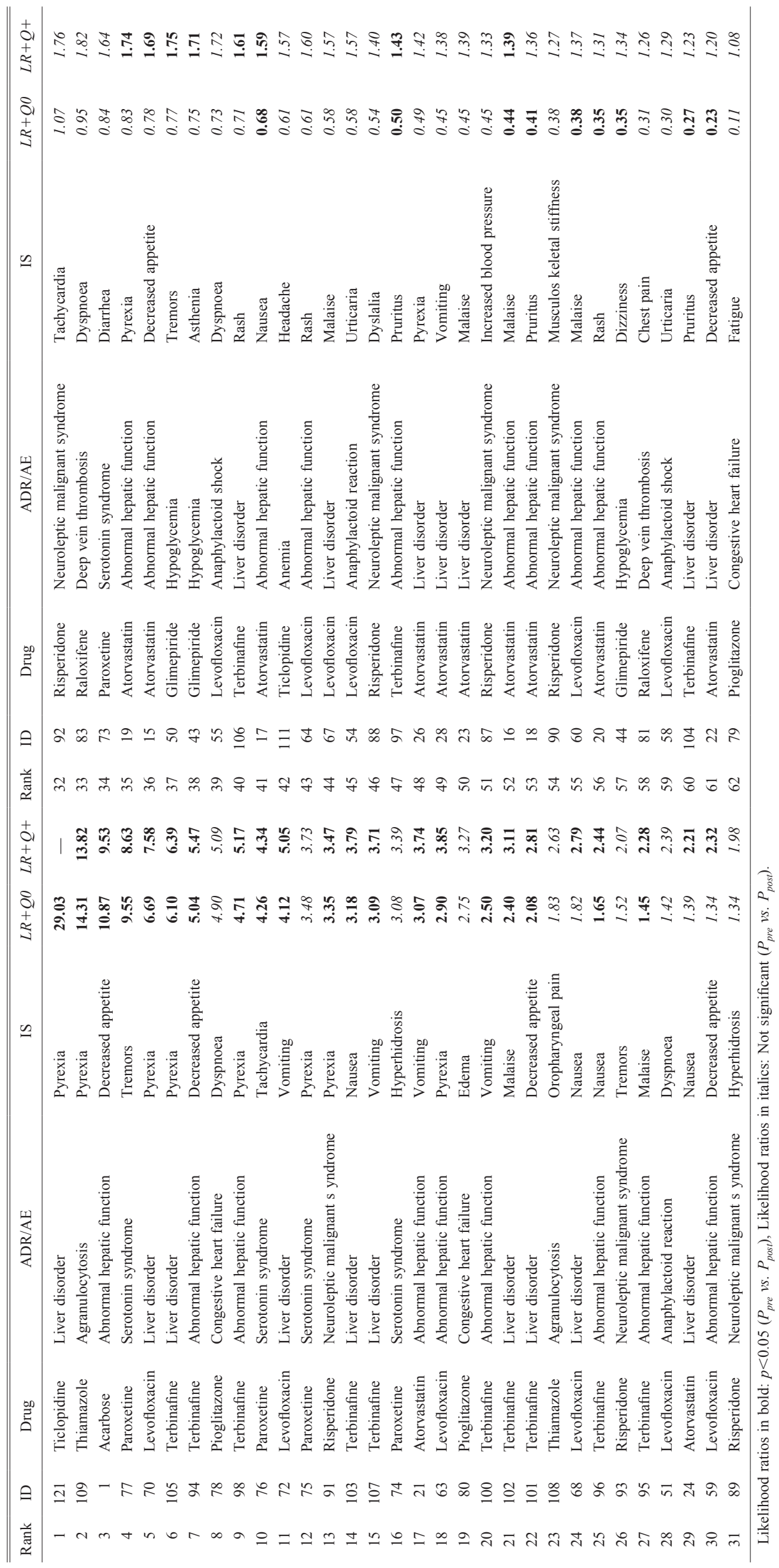


Table 5. Usefulness of IS

\begin{tabular}{|c|c|c|c|c|}
\hline ID & IS & $\begin{array}{l}\text { Listed } \\
(X)\end{array}$ & $\begin{array}{c}\text { Calculated } \\
(Y)\end{array}$ & $\begin{array}{c}\text { Useful } \\
(Z)\end{array}$ \\
\hline 37 & Pyrexia & 15 & 10 & 7 \\
\hline 47 & Vomiting & 9 & 5 & 4 \\
\hline 36 & Decreased appetite & 9 & 6 & 3 \\
\hline 3 & Malaise & 10 & 6 & 2 \\
\hline 30 & Nausea & 10 & 5 & 2 \\
\hline 45 & Tremors & 3 & 3 & 1 \\
\hline 39 & Tachycardia & 2 & 2 & 1 \\
\hline 40 & Dysponea & 6 & 4 & 0 \\
\hline 2 & Pruritus & 11 & 3 & 0 \\
\hline 12 & Rash & 9 & 3 & 0 \\
\hline 33 & Hyperhidrosis & 2 & 2 & 0 \\
\hline 18 & Urticaria & 2 & 2 & 0 \\
\hline 43 & Dyslalia & 4 & 1 & 0 \\
\hline 15 & Oropharyngeal pain & 4 & 1 & 0 \\
\hline 1 & Fatigue & 3 & 1 & 0 \\
\hline 25 & Chest pain & 2 & 1 & 0 \\
\hline 11 & Dizziness & 2 & 1 & 0 \\
\hline 4 & Headache & 2 & 1 & 0 \\
\hline 44 & Asthenia & 1 & 1 & 0 \\
\hline 27 & Increased blood pressure & 1 & 1 & 0 \\
\hline 5 & Diarrhea & 1 & 1 & 0 \\
\hline 32 & Musculoskeletal stiffness & 1 & 1 & 0 \\
\hline 29 & Edema & 1 & 1 & 0 \\
\hline 19 & Palpitations & 5 & 0 & 0 \\
\hline 22 & Hemorrhage $\left(^{*}\right)$ & 3 & 0 & 0 \\
\hline 49 & Hematuria & 1 & 0 & 0 \\
\hline 33 & Somnolence & 1 & 0 & 0 \\
\hline
\end{tabular}

$X$ : number of entries in Table 2, $Y$ : number of drug-ADR-IS for which $L R+$ could be calculated, $Z$ : number of drug-ADR-IS for which $P_{p o s t} Q 0$ and $P_{p o s t} Q+$ were significantly different from $P_{p r e}$ and $L R+Q O>1$. (*) Subcutaneous hemorrhaging + gingival bleeding + epistaxis.

the numbers of ADR cases and ADR accompanied by IS were small.

\section{DISCUSSION}

IS That Were Not Reported to the AERS ( $\star$ ) Some of the ISs listed in the MHD were not reported in the AERS database. In the MHD, the drug-ADR-IS combinations were selected based on their clinical significance. On the other hand, about $40 \%$ of the events in the AERS database were reported by customers. ${ }^{7}$ In addition, most of them were reported after ADR had developed. Therefore, minor changes might have been overlooked in the AERS database, which might have contributed to some of the drug-ADR-IS combinations not being included in the AERS database.

Thus, the IS included in such drug-ADR-IS combinations might be hard to identify by retrospective studies.

ADR/AE That Were Not Found in the AERS Database ( $\star \star)$ No cases of bezafibrate-associated rhabdomyolysis or cibenzoline succinate-associated hypoglycemia were found in the AERS database. It is known that bezafibrate-associated rhabdomyolysis is mainly caused by co-treatment with HMGCoA reductase inhibitors. ${ }^{8)}$ In this study, the AERS search was limited to cases involving monotherapy might explain why no cases were identified. In addition, cibenzoline succinate-associated hypoglycemia frequently develops in patients with renal dysfunction. ${ }^{9)}$ Since most patients with renal dys- function are prescribed multiple drugs, it is reasonable that no cases were identified when the AERS search was limited to monotherapy-treated patients.

In Japan, package inserts are included with all drugs. These inserts are official documents and provide comprehensive information about the drug in question. Since safety is the primary concern during the production of these package inserts, the reporting of not only ADR that occur in the setting of monotherapy but also those that occur during combination treatment anywhere in the world is requested by the Japanese regulatory authority. ${ }^{10)}$ Thus the searching for ADR that occurs in monotherapy setting, using official document focusing on safety, might result in some ADR being missed.

$\boldsymbol{L R}+\boldsymbol{Q 0}$ and $\boldsymbol{L R}+\boldsymbol{Q}+\quad L R+Q+$ was calculated as well as $L R+Q 0$ because it was assumed that the individuals with monotherapy that had reported the AE strongly suspected that the drug in question was the cause of the reported AE, and AE that were not caused by the drug were not included in the database. Accordingly, $Q_{\text {non }}$ was not included in $Q_{\text {pre }}$ or $Q_{\text {other }}$. Thus, we added $Q_{\text {non }}$ in another calculation to set 2 ranges of $P_{\text {post }} ; P_{\text {post }} Q 0$ and $P_{\text {post }} Q+$, to avoid biased conclusion. This procedure also aimed at avoidance of $L R+$ below 1 . That is, the higher the $Q_{n o n}$ to $Q_{\text {pre }}$ ratio, the larger the difference between $L R+Q 0$ and $L R+Q+$; i.e., $L R+Q 0$ is less than 1 and shifts closer to zero, whereas $L R+Q+$ shifts towards 1 .

However, the results of the 2 calculations were mostly the same with regard to the usefulness of the target IS as a predictor of ADR in all calculations, showing that consistent evaluation is possible. Even though a mixture of ADR and AE are included the AERS reports, they might be sufficiently useful to allow the feasibility of IS as predictors of ADR to be evaluated.

Problems Associated with the Use of Useful IS as Predictive Markers In this study, we found that pyrexia and vomiting are useful for predicting severe ADR. However, using such IS could be problematic since telling patients about these potential problems might cause them to become anxious, leading to poor adherence. However, providing information about IS that do not make patients as anxious, such as skin symptoms, including rash and pruritus, and fatigue, might be provided frequently rather than useful IS. Thus, in the clinical setting the information provided to patients about IS should be selected carefully.

Re-calculation of Values Obtained in Our Previous Study $^{1)}$ Using Eq. 2 Equation 2 was derived from Eq. 1, which was newly developed in the present study. Since $Q t$ is included in Eq. 2, it is only applicable when the total number of users of the target drug is known. In our previous study, we utilized a simplified method to obtain $P_{\text {post }} Q^{+}$, and $L R+Q^{+}$ was derived from $P_{\text {post }} Q+{ }^{1)}$ In this study, it was possible to calculate $P_{\text {post }} Q+$ and $L R+Q+$ using Eq. 2. When we recalculated the values obtained in our previous study using Eq. 2, we found that the $L R+Q 0$ and $L R+Q+$ were similar (Table 6).

Data Sources and Study Limitations We used the following variables to perform the calculations in this study: (1) ADR probability values $\left(P_{\text {pre }}\right.$ and $\left.P_{\text {other }}\right)$, (2) the incidences of cases in which the target IS was accompanied by ADR/ AE $\left(Q_{\text {pre }}\right.$ and $\left.Q_{\text {other }}\right)$, and (3) the incidence of I-like symptoms that are not drug-related $\left(Q_{n o n}\right)$. The ADR probability values were obtained from the PMS data reported in drug IF, the IS 
Table 6. Comparison of $P_{\text {post }} Q+$ and $L R+Q+$ Values Derived from Eq. 2 with Those Obtained Using a Simplified Method

\begin{tabular}{|c|c|c|c|c|c|c|}
\hline & \multirow{2}{*}{$P_{\text {pre }}$} & \multicolumn{2}{|c|}{$P_{\text {post }} Q+$} & \multirow{2}{*}{$\begin{array}{c}L R+Q O \\
\text { Previous }\end{array}$} & \multicolumn{2}{|c|}{$L R+Q+$} \\
\hline & & Previous & Eq. 2 & & Previous & Eq. 2 \\
\hline Voriconazole & 0.05000 & 0.04045 & 0.09814 & 5.00 & 0.80 & 2.07 \\
\hline Fenofibrate & 0.00341 & 0.00313 & 0.04512 & 10.82 & 0.92 & 13.79 \\
\hline Sulfametoxazole/trimetoprim & 0.00091 & 0.00329 & 0.01616 & 21.48 & 3.64 & 18.09 \\
\hline Ticlopidine hydrochloride & 0.00176 & 0.00055 & 0.00863 & 2.54 & 0.31 & 4.93 \\
\hline Itraconazole & 0.00081 & 0.00026 & 0.00545 & 4.44 & 0.32 & 6.80 \\
\hline Loxoprofen sodium hydrate & 0.00007 & 0.00017 & 0.00141 & 27.92 & 2.35 & 19.00 \\
\hline
\end{tabular}

incidence data were derived from the AERS database, and the data regarding I-like symptoms that are not drug-related were obtained from an internet-based survey of the general public. Each data source has its own problems.

In Japan, PMS data is collected during clinical practice. Although the resultant PMS data might be affected by selection bias because of the limited number of participating centers and the enrollment of agreeable patient, ${ }^{11)}$ a certain level of quality is assured by the regulatory body since data are collected by pharmaceutical companies under the supervision of the regulatory body. ${ }^{12)}$ Furthermore, as the total number of patients that take a particular drug can be obtained from the PMS data, the incidence of ADR is calculable. ${ }^{12)}$ Therefore we used PMS data for calculating $P_{\text {pre }}$ and $P_{\text {other }}$. However, it was difficult to evaluate the rare ADR, since the sample size was small. Similarly, it was difficult to determine the IS accompanied by ADR from the PMS data. A recent report suggested that small changes in the body condition caused by medication cannot be detected from the PMS data. ${ }^{13)}$ In the present study, we calculated $Q_{\text {pre }}$ and $Q_{\text {other }}$ using AERS data. Since the AERS database is based on a spontaneous reporting system, it has a tendency of underreporting to a greater extent than the abovementioned PMS data. ${ }^{11,14,15)}$ However, the cases in the AERS database involve nearly 5 million subjects, which is a sufficient number to compensate for any such underreporting. On the other hand, since the total number of users of a particular drug cannot be obtained from the AERS database, ADR incidences cannot be derived from it. However, this is not a problem for calculating $Q_{\text {pre }}$ and $Q_{\text {other }}$ since these parameters represent incidence rates among $\mathrm{AE}$ cases associated with the target drug. However, causal relationships have not been demonstrated to exist between the AE reported in the AERS database and the suspected drugs, and some of the reported IS might have been caused by the disease being treated. ${ }^{14)}$ Our results might have been influenced by these factors. Although notoriety bias (due to increased vigilance following safety alerts) has been detected in the AERS databses, ${ }^{16)}$ this might not have influenced the IS incidence data examined in our study, because IS is the mild ADR/AE. Finally, $Q_{\text {non }}$ was obtained from an internet-based survey. Internet-based research (IR) has attracted attention due to the fact that it allows a large amount of data to be obtained cheaply and quickly. Such data are derived from individuals that are registered with research firms. The quality difference (survey results) between research firms is considered to be small ${ }^{17)}$; however, the response distribution patterns of IR are known to differ from those of mail surveys and detention surveys. Some authors have stated that it is wrong to assume that only data obtained by IR deviates from the "true" rate. ${ }^{17)}$ In the present study, $Q_{n o n}$ was obtained from a pre-coded questionnaire. Concerning the causes of any changes in body condition that the IR subjects had experienced during the previous 3 months, the subjects were asked to select: (1) due to an ADR, (2) due to a cause not related to drugs, (3) an unexplained change in body condition that was not related to ADR, or (4) no change in body condition. The probability of selecting (3) was equivalent to $Q_{\text {non }}$. By utilizing a multiple choice answer format, the overreporting of changes in body condition due to an increased awareness of the condition, and hence, more frequent self-diagnosis cannot be ruled out. This is equivalent to the underreporting of ADR incidence data that occurs in spontaneous reports compared with that obtained in observational studies. ${ }^{15)}$ Thus, $Q_{\text {non }}$ might overestimate the "true" probability. Accordingly, this could result in $L R+Q 0$ values of less than 1 for ADR with small $Q_{\text {pre }}$ values, as well as the underestimation of both $P_{\text {post }} Q^{+}$ and $L R+Q+$.

Future Subjects This study focused on drug-ADRIS combinations that the pharmacists considered clinically important. Thus, other drug-ADR-IS combinations that are encountered in clinical practice remain to be examined. It is necessary to exhaustively investigate useful IS in order to be able to predict ADR.

Ghajar et al. investigated the usefulness of flare as an IS for predicting sulfonamide-induced drug eruption based on the type and onset time of the flare using Bayes' theorem. ${ }^{18)}$ It is necessary to modify calculations such as ours in order to increase the probability of prevention, e.g., by adding information concerning the onset time of IS. It is also necessary to investigate the usefulness of evaluating IS in consideration of the concomitant use of multiple drugs because ADR overlap and the probability of an ADR developing alters when multiple drugs are used concomitantly. ${ }^{19)}$

\section{CONCLUSION}

By using the AERS database, we were able to quantitatively evaluate a markedly increased number of drug-ADR-IS combinations using Bayes' theorem. As a result, it was suggested that fever, nausea, and decreased appetite are useful predictors of ADR, but pruritus and rash are less useful.

For thiamazole-induced agranulocytosis and levofloxacinor terbinafine-induced liver disorder, fever might be a useful predictor of ADR; tremors might be useful for detecting paroxetine-induced serotonin syndrome; and decreased appetite might be a useful indicator of terbinafine-induced liver dysfunction. 


\section{REFERENCES}

1) Ohshima S, Ieda M, Yamamoto M, Kobayashi D. Quantitative evaluation method of information value of initial symptoms based on Bayesian theory. Yakugaku Zasshi, 132, 763-768 (2012).

2) Scandellari C. The Bayesian approach to evaluation of diagnostic data. Ann. Ist. Super. Sanita, 27, 385-393 (1991).

3) Ministry of Health, Labour and Welfare. "the Manual for Handling Disorders due to Adverse Drug Reactions.": 〈http://www.mhlw. go.jp/topics/2006/11/tp1122-1.html), cited 6 May, 2013.

4) Saito M. Introduction of "the manual for handling disorders due to adverse drug reactions": focus on the antibiotics related severe adverse drug reactions. Jpn. J. Antibiot., 61, 269-288 (2008).

5) Jose J, Rao PG. Pattern of adverse drug reactions notified by spontaneous reporting in an Indian tertiary care teaching hospital. Pharmacol. Res., 54, 226-233 (2006).

6) Colloca L, Finniss D. Noceboeffects, patient-clinician communication, and therapeutic outcomes. JAMA, 307, 567-568 (2012).

7) U.S. Food and Drug Administration. "AERS Reporting by Healthcare Providers and Consumers by Year (As of December 31, 2010).”: 〈http://www.fda.gov/Drugs/GuidanceComplianceRegulatoryInformation/Surveillance/AdverseDrugEffects/ucm070456.htm>, cited 10 May, 2013.

8) Wu J, Song Y, Li H, Chen J. Rhabdomyolysis associated with fibrate therapy: review of 76 published cases and a new case report. Eur. J. Clin. Pharmacol., 65, 1169-1174 (2009).

9) Interview form of cibenzoline succinate tablets, cited 11 May, 2013.

10) Ministry of Health, Labour and Welfare. "Instructions for Descrip- tion on Drug Usages" (the 607th Notification from the Pharmaceutical Affairs Bureau issued on April 25th, 2007).

11) Ohashi Y. Progress report of the working group on "future postmarketing surveillance (PMS) in Japan." Jpn. J. Pharmacoepidemiol., 8, 1-35 (2003).

12) Watanabe S, Nakano Y, Nomura K. Steps in developing a database of drug use-result surveillance: as an example of anti-hyperlipidemia drugs. Jpn. J. Pharmacoepidemiol., 17, 87-97 (2012).

13) Gnädinger M, Mellinghoff HU. The out-of-focus bias in drug surveillance. Eur. J. Clin. Pharmacol., 69, 357-359 (2013).

14) Fujita T. Signal detection of adverse drug reactions. Jpn. J. Pharmacoepidemiol., 14, 27-36 (2009).

15) Fletcher AP. Spontaneous adverse drug reaction reporting $v s$. event monitoring: a comparison. J. R. Soc. Med., 84, 341-344 (1991).

16) Raschi E, Piccinni C, Poluzzi E, Marchesini G, Ponti F D, The association of pancreatitis with antidiabetic drug use: gaining insight through the FDA pharmacovigilance database, Acta Diabetol: Published on line 19 October 2011.

17) Developing reliable internet-based surveys, SSJDA-42.: 〈https:// ssjda.iss.u-tokyo.ac.jp/rps/RPS042.pdf), cited 11 May, 2013.

18) Ghajar BM, Lanctôt KL, Shear NH, Naranjo CA. Bayesian differential diagnosis of a cutaneous reaction associated with the administration of sulfonamides. Semin. Dermatol., 8, 213-218 (1989).

19) Endo T, Watanabe N, Goto K, Kutsuma N, Komada F, Saitoh Y, Kobayashi D, Morimoto Y. Overlapping of the clinically significant adverse reaction of drugs in the polypharmacy. Jpn. J. Pharm. Health Care Sci., 28, 157-163 (2002). 